\section{Percepción de la desviación de la línea media de la sonrisa por individuos no relacionados a la Odontología y por especialistas en Ortodoncia}

\author{
Perception of midline deviation by \\ laypersons and orthodontists
}

\begin{abstract}
Resumen
Objetivo: Comparar la percepción de la desviación de la línea media de la sonrisa por individuos no relacionados a la Odontología y ortodoncistas. Materiales y método: Estudio descriptivo y transversal; en el que participaron 102 personas, 60 no relacionados a la odontología y 42 ortodoncistas, quienes calificaron la estética de la sonrisa en fotografías según la posición de la línea media, usando una escala visual análoga de 0-100 mm; para ello se modificó la fotografía de sonrisa de un paciente varón, de 17 años, en oclusión óptima, simétrico, con posición de línea media de la sonrisa centrada, creándole desviaciones a la derecha e izquierda, usando el programa Adobe Photoshop CS6. Resultados: Para ambos grupos de evaluadores, la percepción de las desviaciones de la línea media de la sonrisa de 1, 2, 3 y $4 \mathrm{~mm}$ hacia la derecha y de $1 \mathrm{~mm}$ hacia la izquierda fueron estadísticamente significativos $(\mathrm{p}<0,05)$ y los resultados de las desviaciones hacia la izquierda de $2,3$ y $4 \mathrm{~mm}$ y posición centrada no fueron estadísticamente significativos ( $\mathrm{p}>0,05)$. Se encontraron diferencias en las percepciones según el lado para el cual se encuentran éstas desviaciones. Conclusiones: Los ortodoncistas fueron más críticos al mostrar la percepción de la desviación de la línea media de la sonrisa en comparación con los individuos no relacionados a la odontología, lo que se evidencia en la menor tolerancia al percibir la estética de las desviaciones mayores de línea media de sonrisa.
\end{abstract}

Palabras clave: Escala visual analógica; Ortodoncia; Percepción; Sonrisa.

\begin{abstract}
Objective: To compare the perception of the smile midline deviation by laypersons and orthodontists. Material and method: Descriptive and cross-sectional study; in which 102 subjects participated, 60 laypersons and 42 orthodontists, who evaluated the aesthetics of the smile in photos according to the position of the midline, using a visual analog scale from 0-100 mm; for this, a smile photograph of a 17 years old male patient, with optimal occlusion, symmetrical smile midline position centered was modified by creating deviations to the right and left, using the program Adobe Photoshop CS6. Results: For both evaluators, the results for the deviations of $1,2,3$ and $4 \mathrm{~mm}$ to the right and $1 \mathrm{~mm}$ to the left were statistically significant $(\mathrm{p}<0.05)$ and the results for deviations to the left of 2,3 and $4 \mathrm{~mm}$ and centered position groups were not significant $(\mathrm{p}>0.05)$. There are differences in perceptions according to the side where are the deviations. Conclusion: Orthodontists were more critical to show the perception of the aesthetics of the midline deviations compared with laypersons.
\end{abstract}

Keywords: Orthodontics; Perception; Smile; Visual analog scale.

\section{Artículo Original}

Sandra Victoria Cabello-Pérez ${ }^{1, a}$, Luciano Carlos Soldevilla-Galarza ${ }^{1, a, b}$, Ney Alberto Paredes-Sampén ${ }^{1, a, b}$

${ }^{1}$ Facultad de Odontología. Universidad Nacional Mayor de San Marcos. Lima, Perú.

a Cirujano Dentista

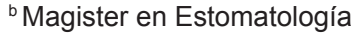

\section{Correspondencia:}

Sandra Victoria Cabello-Pérez

Correo electrónico: sandravictoriacabelloperez@ gmail.com

Av. Germán Amezaga Nº 375 - Ciudad Universitaria - Lima 1, Perú.

\section{Coautores:}

Luciano Carlos Soldevilla-Galarza

lucianosol@yahoo.com

Ney Alberto Paredes-Sampén

neydent@hotmail.com

Conflicto de intereses: Los autores declaran no tener conflictos de interés.

Fuente de financiamiento: Autofinanciado.

Fecha de recepción: 15/05/17

Fecha de aceptación: 10/11/17 


\section{Introducción}

La estética puede ser definida como el reflejo de una apariencia agradable. Peck y Peck ${ }^{1}$ definieron la estética como la apreciación de verse bien o percepción de la belleza, por lo tanto, la estética y la belleza están relacionadas con las sensaciones de agrado que un individuo tiene con respecto a las cualidades de una característica en particular. Así, el concepto de belleza es único para cada persona y es establecido basándose en factores como el género, raza, educación y ocupación, entre otros. Tener una sonrisa hermosa y agradable es el principal consenso entre los pacientes de ortodoncia y se evidencia en el aumento de la demanda de tratamientos estéticos en las consultas odontológicas. Chang et al. ${ }^{2}$ sostienen que una sonrisa atractiva es un elemento clave en la satisfacción de los pacientes ortodónticos. Una sonrisa bella inspira atracción e influye en la primera impresión personal del individuo.

Un elemento importante en la estética facial es la línea media de la sonrisa (considerada dentro de la macroestética según la clasificación de Sarver ${ }^{3}$ ), que se define como la línea vertical imaginaria formada por la unión de los dos incisivos centrales superiores, que idealmente coincide con la línea media facial. La posición de la línea media de la sonrisa es importante para una sonrisa agradable y la propia satisfacción de la persona. Generalmente los tratamientos ortodónticos finalizan con posiciones ideales de esta línea media, pero existen situaciones donde es difícil conseguir este objetivo.

Estudiar la percepción estética es una acción compleja, ya que ésta difiere de persona a persona, es un aspecto subjetivo al que se le debe dar objetividad, a pesar que la percepción varíe por influencia de ciertos factores culturales y generacionales, principalmente. Mc Leod et al. ${ }^{4}$ encontraron que se presentan diferencias culturales relacionadas a las características de la sonrisa. Existen investigaciones que evaluaron el criterio estético de la percepción de la desviación de la línea media usando fotografías de sonrisa modificadas intencionalmente con una computadora y sometiéndolas a evaluación por uno, dos o tres grupos (ortodoncistas, pacientes y odontólogos generales). Williams ${ }^{5}$ encontró que el tipo facial y el género pueden afectar los umbrales en los que las desviaciones de línea media se detectan y por encima del cual la desviación es considerada inaceptable.

La desviación de la línea media es considerada como un factor antiestético de la sonrisa. Los antecedentes refieren aceptación de desviaciones de sonrisa de 2 a $3 \mathrm{~mm}$ ${ }^{5-8}$. Es importante conocer la magnitud de la desviación y saber si ésta puede ser percibida por una persona no relacionada a la odontología y por un ortodoncista como un factor antiestético?

El objetivo del estudio fue comparar la percepción de la desviación de la línea media de la sonrisa por individuos no relacionados a la odontología (sin estudios odontológicos ni vinculados laboralmente con la odontología) y por ortodoncistas (expertos en reconocimientos de asimetrías).

\section{Materiales y método}

Estudio descriptivo y transversal. La población estuvo constituida por personas no relacionadas a la odontología o sin conocimientos específicos de esta profesión, de ambos géneros, durante el año 2015, y por ortodoncistas calificados sin límites de edad ni género. La determinación del tamańo de la muestra para cada uno de los dos grupos se realizó aplicando la fórmula para tamaño muestral para comparar proporciones con marco muestral conocido, obteniendo 60 individuos para el grupo de los no relacionados a la odontología y 42 para los ortodoncistas; el nivel de confianza usado fue de $97,5 \% \mathrm{La}$ muestra fue aleatoria simple para el grupo de individuos no relacionados a la odontología, quienes eran alumnos de la Escuela Académico Profesional de Educación Semi Escolarizada (EAPESE) de la Facultad de Educación de la Universidad Nacional José Faustino Sánchez Carrión. La conformación de la muestra para el grupo de ortodoncistas fue por casos consecutivos, se incluyeron ortodoncistas calificados de diferentes facultades de Odontología. Fueron excluidas las personas que hayan tenido información ortodóntica previa (pacientes o expacientes de tratamientos de ortodoncia) y estudiantes de odontología, tanto de pregrado como de posgrado.

El método empleado fue la encuesta, se usó un instrumento en formato de catálogo fotográfico y fichas para el llenado de datos generales y calificación del individuo encuestado, previamente validado por expertos. La encuesta presentó dos componentes: datos personales y datos propios de la investigación.

Se utilizó la fotografía de sonrisa de un paciente varón, de 17 años, en oclusión óptima, con simetría facial transversal y con proporciones adecuadas de los tercios faciales superior, medio e inferior, tomada con una cámara Canon Rebel T3I, con lente macro 100 mm f/ 1.4 y ringflash, con línea media de la sonrisa en posición centrada, la cual se modificó con el programa Adobe Photoshop CS6 hasta lograr desviaciones a la derecha e izquierda de 1,2, 3 y $4 \mathrm{~mm}$ (Figura 1). Se empleó en este caso la escala visual análoga $0-100 \mathrm{~mm}$ en cada fotografía 10, protegidas por micas transparentes y archivadas en un folder plastificado. Así mismo se requirió un plumón negro o azul punta fina no indeleble, regla milimetrada, algodón y un frasco pequeño de alcohol.

Los participantes fueron informados del objetivo de la investigación (se les indicó que debían evaluar la desviación de la línea media de la sonrisa, en el caso de los individuos no relacionados a la odontología se les hizo una breve explicación del concepto) y a las personas que cumplieron con los criterios de inclusión se les solicitó el consentimiento y sus datos. Se les pidió que califiquen la estética de la sonrisa del paciente en una escala visual análoga 0-100 mm colocada en la parte inferior de la fotografía según las categorías: muy estético, estético, aceptable, desagradable o muy desagradable, siendo el lado izquierdo (cercano a 0) muy desagradable, la zona contigua hacia la derecha como desagradable, la zona del medio (50) aceptable, la zona contigua como estético y la zona de la derecha (cercano a 100) muy agradable a la 


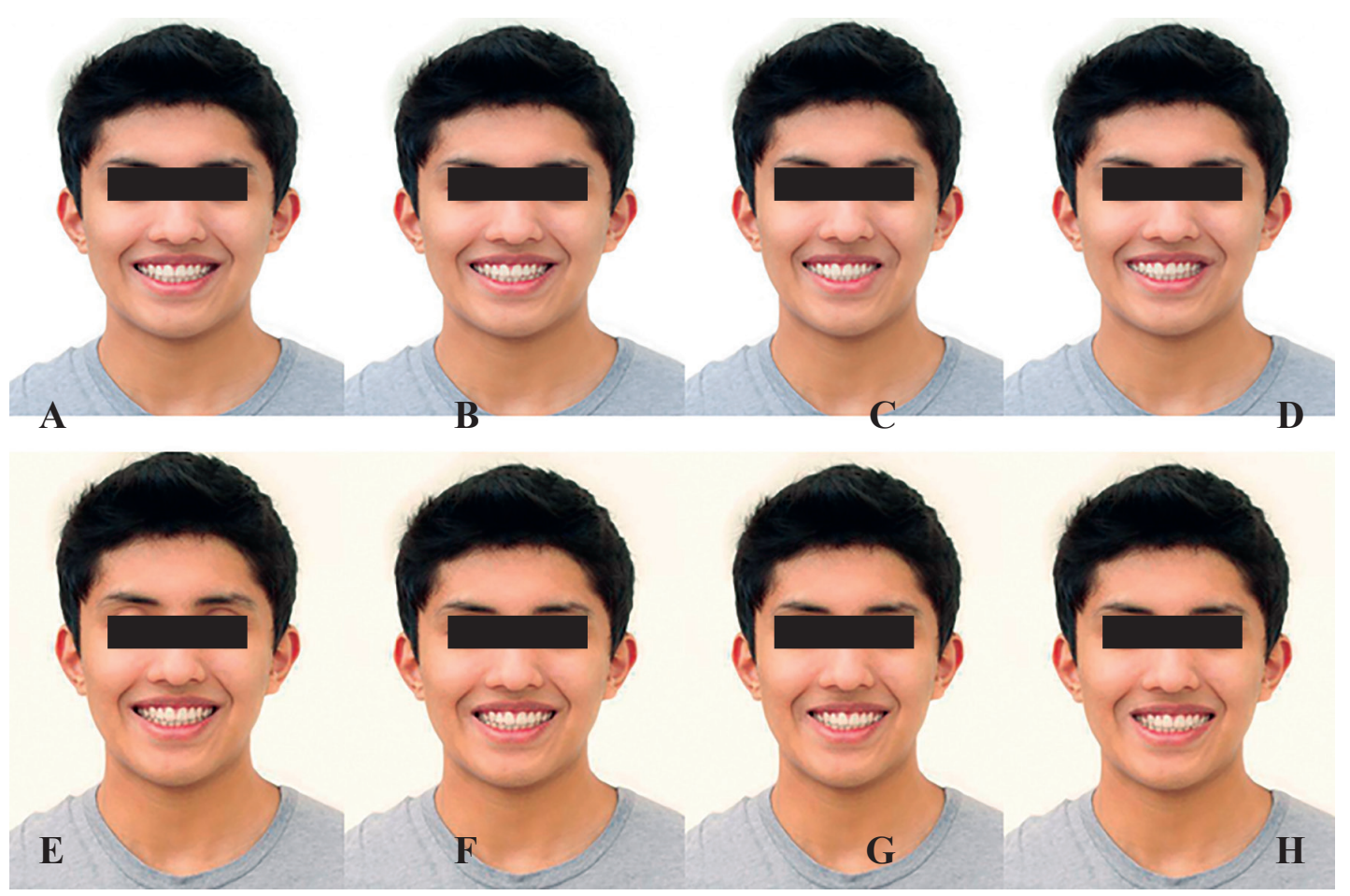

Figura 1. Desviaciones de línea media de la sonrisa creadas a partir de la fotografía de un paciente simétrico. A) $1 \mathrm{~mm}$ a la derecha. B) $2 \mathrm{~mm}$ a la derecha. C) $3 \mathrm{~mm}$ a la derecha. D) $4 \mathrm{~mm}$ a la derecha. E) $1 \mathrm{~mm}$ a la izquierda. F) $2 \mathrm{~mm}$ a la izquierda. G) $3 \mathrm{~mm}$ a la izquierda. H) $4 \mathrm{~mm}$ a la izquierda.

vista o muy estético para el encuestado. Previamente se les explicó el procedimiento en fotografías de otros casos y observaron rápidamente cada una de las fotografías a evaluar durante 5 segundos para familiarizarse con las ellas y no fatigar la visión y evitar falsear la calificación. El encuestado marcó mediante un trazo perpendicular a la línea horizontal de la escala el lugar donde consideraba adecuado. Las fotografías se ordenaron aleatoriamente. Las calificaciones exactas se obtuvieron al medir la ubicación de la marca realizada con el plumón punta fina en milímetros con ayuda de la regla milimetrada y se registró en la ficha de recolección de datos, ya que el trazo de la escala midió $100 \mathrm{~mm}$ y registró, análogamente, de 0 a 100 puntos. Para fines de la calificación se consideró 0-20: muy desagradable, 21-40: desagradable, 41-60: aceptable, 61-80: estético y 81-100: muy estético 11. Para utilizar el instrumento en los demás individuos se protegieron las fotografías en micas transparentes y se limpiaban las marcas con un algodón con alcohol. De la misma manera se realizó la encuesta fotográfica en los ortodoncistas.

El procesamiento y análisis de datos se realizó haciendo uso del programa IBM SPSS Statistics 22.0. Para la descripción de las variables se utilizaron tablas de frecuencia, valores de medianas para cada categoría y pruebas estadísticas U de Mann-Whitney. El nivel de significancia aplicado fue de 0,05.

\section{Resultados}

El grupo de individuos no relacionados a la odontología estuvo conformado por $80 \%$ mujeres y $20 \%$ varones mientras que los ortodoncistas fueron $66,7 \%$ varones y 33,3\% mujeres. El grupo de individuos no relacionados a la odontología estuvo conformado por $63,3 \%$ adultos jóvenes (entre 18 a 39 años de edad) y 36,7\% adultos maduros (entre 40 a 60 años de edad) y los ortodoncistas fueron 31\% adultos jóvenes y $69 \%$ adultos maduros. Las pruebas de normalidad indicaron aplicar pruebas no paramétricas. Como medida de tendencia central se tomaron las medianas de la percepción de la desviación para ambos grupos. Se compararon los resultados obtenidos entre cada grupo evaluador con respecto a cada situación de posición de línea media mediante tablas indicando frecuencias y porcentajes y prueba $U$ de Mann-Whitney (Tabla 1).

Las desviaciones de la línea media hacia el lado derecho tienen mayores porcentajes de no aceptación en sus cuatro versiones y en ambos grupos evaluadores (Tabla 2).

Por el contrario, las desviaciones hacia la izquierda tuvieron mayor aceptación en sus cuatro versiones en los individuos no relacionados a la odontología; en el grupo de ortodoncistas hubo diversidad de resultados porcentuales en la evaluación de la percepción (Tabla 3).

En el caso ideal, de línea media de la sonrisa centrada, ambos grupos evaluadores califican mayoritariamente con aceptación las percepciones estéticas de la sonrisa del modelo (Tabla 4).

Para ambos grupos de evaluadores, los resultados de las desviaciones de la línea media de la sonrisa de 1, 2, 3 y $4 \mathrm{~mm}$ hacia la derecha y de $1 \mathrm{~mm}$ hacia la izquierda fueron estadísticamente significativos $(\mathrm{p}<0,05)$ y los resultados de las desviaciones hacia la izquierda de 2, 3 y $4 \mathrm{~mm}$ y posición centrada fueron estadísticamente no 
significativos $(\mathrm{p}>0,05)$. Las percepciones de las desvia- izquierdo, siendo las desviaciones hacia el lado izquierdo ciones hacia el lado derecho difieren, para las mismas las mejor toleradas por ambos grupos evaluadores ( $\mathrm{Ta}$ magnitudes de desviación, con respecto de las del lado blas 2-4).

Tabla 1. Estadísticos de percepción de la desviación de la línea media de sonrisa

\begin{tabular}{|c|c|c|c|c|c|c|c|c|c|c|}
\hline \multirow{2}{*}{$\begin{array}{l}\text { Análisis } \\
1 \mathrm{~mm}\end{array}$} & & \multicolumn{4}{|c|}{ Desviación hacia la derecha } & \multirow{2}{*}{$\begin{array}{l}\text { Posición } \\
\text { centrada } \\
1 \mathrm{~mm}\end{array}$} & \multicolumn{4}{|c|}{ Desviación hacia la izquierda } \\
\hline & & $2 \mathrm{~mm}$ & $3 \mathrm{~mm}$ & $4 \mathrm{~mm}$ & & & $2 \mathrm{~mm}$ & $3 \mathrm{~mm}$ & $4 \mathrm{~mm}$ & \\
\hline \multirow[t]{2}{*}{ Mediana* } & $\begin{array}{l}\text { Individuos no } \\
\text { relacionados a } \\
\text { la odontología }\end{array}$ & 2 & 2 & 2 & 1 & 3 & 3 & 2 & 2 & 2 \\
\hline & Ortodoncistas & 1 & 1 & 0 & 0 & 3 & 2 & 3 & 3 & 2 \\
\hline \multicolumn{2}{|c|}{ U de Mann Whitney } & 781,0 & 507,0 & 386,5 & 506,0 & 1134,5 & 956,0 & 1108,0 & 1019,5 & 1130,0 \\
\hline \multicolumn{2}{|c|}{ Significancia } & 0,001 & $<0,001$ & $<0,001$ & $<0,001$ & 0,380 & 0,033 & 0,283 & 0,091 & 0,360 \\
\hline
\end{tabular}

* Los valores de las medianas corresponden a 0) "muy desagradable", 1) "desagradable",

2) “aceptable", 3) "estético", 4) "muy estético"

Tabla 2. Percepción de desviación de línea media de la sonrisa hacia la derecha

\begin{tabular}{|c|c|c|c|c|c|c|c|c|c|c|c|c|c|c|c|c|}
\hline \multirow{3}{*}{$\begin{array}{l}\text { Percepción de } \\
\text { desviación a la } \\
\text { derecha }\end{array}$} & \multicolumn{8}{|c|}{ No relacionados a la odontología } & \multicolumn{8}{|c|}{ Ortodoncistas } \\
\hline & \multicolumn{2}{|c|}{$1 \mathrm{~mm}$} & \multicolumn{2}{|c|}{$2 \mathrm{~mm}$} & \multicolumn{2}{|c|}{$3 \mathrm{~mm}$} & \multicolumn{2}{|c|}{$4 \mathrm{~mm}$} & \multicolumn{2}{|c|}{$1 \mathrm{~mm}$} & \multicolumn{2}{|c|}{$2 \mathrm{~mm}$} & \multicolumn{2}{|c|}{$3 \mathrm{~mm}$} & \multicolumn{2}{|c|}{$4 \mathrm{~mm}$} \\
\hline & $\mathbf{n}$ & $\%$ & $\mathbf{n}$ & $\%$ & $\mathbf{n}$ & $\%$ & $\mathbf{n}$ & $\%$ & $\mathbf{n}$ & $\%$ & $\mathbf{n}$ & $\%$ & $\mathbf{n}$ & $\%$ & $\mathbf{n}$ & $\%$ \\
\hline Muy desagradable & 2 & 3,3 & 2 & 3,3 & 2 & 3,3 & 8 & 13,3 & 11 & 26,2 & 14 & 33,3 & 24 & 57,1 & 27 & 64,3 \\
\hline Desagradable & 13 & 21,7 & 23 & 38,3 & 25 & 41,7 & 27 & 45 & 11 & 26,2 & 21 & 50 & 14 & 33,3 & 12 & 28,6 \\
\hline Aceptable & 18 & 30 & 10 & 16,7 & 15 & 25 & 12 & 20 & 13 & 31 & 7 & 16,7 & 3 & 7,1 & 2 & 4,8 \\
\hline Estético & 15 & 25 & 16 & 26,7 & 10 & 16,7 & 9 & 15 & 1 & 2.4 & 0 & 0 & 0 & 0 & 0 & 0 \\
\hline Muy Estético & 12 & 20 & 9 & 15 & 8 & 13,3 & 4 & 6,7 & 6 & 14,3 & 0 & 0 & 1 & 2,4 & 1 & 2,4 \\
\hline Total & 60 & 100 & 60 & 100 & 60 & 100 & 60 & 100 & 42 & 100 & 42 & 100 & 42 & 100 & 42 & 100 \\
\hline
\end{tabular}

Tabla 3. Percepción de desviación de línea media de la sonrisa hacia la izquierda

\begin{tabular}{|c|c|c|c|c|c|c|c|c|c|c|c|c|c|c|c|c|}
\hline \multirow{3}{*}{$\begin{array}{l}\text { Percepción de } \\
\text { desviación a la } \\
\text { izquierda }\end{array}$} & \multicolumn{8}{|c|}{ No relacionados a la odontología } & \multicolumn{8}{|c|}{ Ortodoncistas } \\
\hline & \multicolumn{2}{|c|}{$1 \mathrm{~mm}$} & \multicolumn{2}{|c|}{$2 \mathrm{~mm}$} & \multicolumn{2}{|c|}{$3 \mathrm{~mm}$} & \multicolumn{2}{|c|}{$4 \mathrm{~mm}$} & \multicolumn{2}{|c|}{$1 \mathrm{~mm}$} & \multicolumn{2}{|c|}{$2 \mathrm{~mm}$} & \multicolumn{2}{|c|}{$3 \mathrm{~mm}$} & \multicolumn{2}{|c|}{$4 \mathrm{~mm}$} \\
\hline & $\mathbf{n}$ & $\%$ & $\mathbf{n}$ & $\%$ & $\mathbf{n}$ & $\%$ & $\mathbf{n}$ & $\%$ & $\mathbf{n}$ & $\%$ & $\mathbf{n}$ & $\%$ & $\mathbf{n}$ & $\%$ & $\mathbf{n}$ & $\%$ \\
\hline Muy desagradable & 1 & 1,7 & 3 & 5 & 3 & 5 & 2 & 3,3 & 4 & 9,5 & 1 & 2,4 & 1 & 2,4 & 3 & 7,1 \\
\hline Desagradable & 11 & 18,3 & 12 & 20 & 13 & 21,7 & 15 & 25 & 11 & 26,2 & 9 & 21,4 & 8 & 19 & 14 & 33,3 \\
\hline Aceptable & 15 & 25 & 18 & 30 & 19 & 31,7 & 22 & 36,7 & 13 & 31 & 10 & 23,8 & 9 & 21,4 & 11 & 26,2 \\
\hline Estético & 17 & 28,3 & 22 & 36,7 & 18 & 30 & 8 & 13,3 & 6 & 14,3 & 13 & 31 & 13 & 31 & 6 & 14,3 \\
\hline Muy Estético & 16 & 26,7 & 5 & 8,3 & 7 & 11,7 & 13 & 21,7 & 8 & 19 & 9 & 21,4 & 11 & 26,2 & 8 & 19 \\
\hline Total & 60 & 100 & 60 & 100 & 60 & 100 & 60 & 100 & 42 & 100 & 42 & 100 & 42 & 100 & 42 & 100 \\
\hline
\end{tabular}


Percepción de la desviación de la línea media de la sonrisa por individuos no relacionados a la Odontología...

Tabla 4. Percepción de línea media de la sonrisa en posición centrada

\begin{tabular}{lllll}
\hline \multirow{2}{*}{$\begin{array}{l}\text { Percepción de línea media } \\
\text { centrada }\end{array}$} & \multicolumn{2}{l}{ No relacionados a la odontología } & \multicolumn{2}{l}{ Ortodoncistas } \\
\cline { 2 - 5 } & $\mathbf{n}$ & $\mathbf{\%}$ & $\mathbf{n}$ & $\mathbf{\%}$ \\
\hline Muy Desagradable & 0 & 0 & 6 & 14,3 \\
Desagradable & 15 & 25 & 7 & 16,7 \\
Aceptable & 12 & 20 & 7 & 16,7 \\
Estético & 16 & 26,7 & 12 & 28,8 \\
Muy Estético & 17 & 28,3 & 10 & 23,8 \\
\hline Total & 60 & 100 & 42 & 100 \\
\hline
\end{tabular}

\section{Discusión}

El presente estudio, a diferencia de los reportados previamente, incluyó entre sus categorías la dirección de la desviación: lado izquierdo y lado derecho. De los resultados obtenidos, se establecen diferencias entre las percepciones estéticas obtenidas de las desviaciones de línea media de la sonrisa hacia la derecha con respecto a las desviaciones de línea media de la sonrisa hacia la izquierda. Se encontró que las desviaciones hacia la izquierda son mejor toleradas que aquellas presentes en el lado derecho. Al revisar publicaciones con respecto a la línea media de la sonrisa se encontró que existen algunos que tan solo evaluaron la prevalencia de ésta ${ }^{12}$ y sus desviaciones en determinadas poblaciones, otros evaluaron la cantidad de desviación observable por una muestra determinada ${ }^{7}$, otros evaluaron tan solo si las desviaciones eran observables o no ${ }^{13}$, y otros estudios evaluaron la percepción estética de las desviaciones de línea media ${ }^{7,8,14}$, es decir, le agregaron una calificación a la evaluación de la percepción del individuo observador.

En el 2003 Cardash ${ }^{15}$ determinó la desviación observable de línea media, se enfocó solamente en la detección de esta situación mas no en la percepción estética, usó una muestra de 10 observadores y encontró que el 83\% recién detecta las desviaciones cuando son mayores a 2 mm y el 37\% las detecta cuando son de 1 a $2 \mathrm{~mm}$; para el valor de desviación de $1 \mathrm{~mm}$ este estudio obtuvo resultados de aceptabilidad en un rango de $28,3 \%$ a $31 \%$, lo que podría indicar que este porcentaje de evaluadores posiblemente no ha percibido la desviación a $1 \mathrm{~mm}$.

En el 2006 Kokich ${ }^{14}$ evaluó la percepción del diastema presente a nivel de la línea media interdental en la arcada superior en la que agregó a odontólogos generales como tercer grupo. De la misma manera empleó una escala visual análoga para evaluar la percepción, pero en este caso de $50 \mathrm{~mm}$ y la escala fue: menos atractivo más atractivo. En cuanto a los grupos evaluadores, fueron los ortodoncistas más críticos al evaluar. Siguiendo un procedimiento similar al de este estudio, en el 2007, Pinho ${ }^{9}$ evaluó la percepción estética de la desviación de la línea media manipulando fotografías y agregando un tercer grupo de protesistas. $\mathrm{Al}$ igual que en el presente estudio, usó una escala visual análoga de $100 \mathrm{~mm}$ y rea- lizó desviaciones de hasta $4 \mathrm{~mm}$, pero tan solo hacia el lado derecho. Pinho encontró que las personas comunes no identificaron las desviaciones, los protesistas las identificaron a partir de los $3 \mathrm{~mm}$ y los ortodoncistas a partir de $1 \mathrm{~mm}$ de desviación.

Tupinambá ${ }^{8}$, en el 2009, encontró aceptable las desviaciones de $3 \mathrm{~mm}$. Para su estudio empleó la fotografía de un individuo, una escala de 0 a 10 puntos (menos atractivo- más atractivo) y realizó las alteraciones de línea media hasta $3 \mathrm{~mm}$, es decir la máxima desviación presentada fue considerada aceptable por su muestra. En este estudio la máxima desviación presentada no fue tolerada.

Springer ${ }^{7}$, en el 2010, modificó la línea media dental de la arcada superior hacia la izquierda, teniendo a la línea media facial y al filtrum como guía, y encontró aceptable las desviaciones de $3,2 \mathrm{~mm}$ en promedio, lo que coincide en cierta forma con los resultados de esta investigación, ya que para las desviaciones de $3 \mathrm{~mm}$ hacia la izquierda se encontró que los individuos no relacionados a la odontología la perciben como aceptable y los ortodoncistas, incluso, la perciben como estética. Esto podría deberse a ciertos fundamentos de psicología que estudian la percepción y el cómo los seres humanos miran y perciben un objeto visual, para lo cual se debe tener presente el concepto de peso visual. Se dice que un objeto pesa menos en el lado izquierdo del cuadro (a la altura de la derecha del observador) y, a la vez, que el lado izquierdo soporta más peso que el derecho, soportar más peso significa que las cargas soportadas, siendo las mismas, se aligeran de parte de su densidad visual, es decir, pesan menos ${ }^{16}$, lo que justificaría el porqué, las desviaciones presentadas en el lado derecho de la fotografía que se está observando son mejor toleradas que las del lado opuesto, y este fundamento se plasma en los resultados del presente estudio. Así mismo, es necesario mencionar que también influye la cultura de los evaluadores, principalmente el tipo de escritura: en poblaciones occidentales, la escritura se realiza en dirección de izquierda a derecha, lo que acostumbra la visión a ir en ese mismo sentido ${ }^{17}$ y también interviene la lateralidad del individuo, es decir si es diestro o zurdo, ya que los diestros tienen mayor desarrollo del hemisferio izquier- 
do; para este estudio no se consideraron estos aspectos de escritura y lateralidad en vista de que no se tenía el hallazgo de las consecuencias e influencias del peso visual respecto del lado de presentación de la desviación antes de ejecutar la investigación, podría ser un aspecto importante a considerar investigar en el futuro.

Existe variación en la percepción respecto del género del modelo al cual se le realizan las modificaciones. Zhang ${ }^{11}$, en el 2010 en su estudio de percepción según el género y la cara encontró menor tolerancia a las desviaciones en modelos femeninos que en los masculinos, las evaluadoras fueron más tolerantes en desviaciones de modelos masculinos; el presente estudio tuvo uno de los grupos evaluadores conformado por un $80 \%$ de mujeres (no relacionadas a la odontología) y el sujeto evaluado fue masculino, y este grupo evaluador de mayoría femenina brindó mayor tolerancia a las desviaciones en el único modelo (masculino). Existen revisiones sistemáticas, de Witt ${ }^{18}$ y de Janson ${ }^{6}$, en el 2011, que hallaron que la mayoría de las personas comunes son capaces de detectar desviaciones de $3 \mathrm{~mm}$, pero en cuanto a preferencias eligen las líneas medias coincidentes. Janson ${ }^{6}$ en su revisión obtuvo el valor de 2,2 $\mathrm{mm}$ de promedio aceptado de desviación de línea media por personas comunes y ortodoncistas; en el presente estudio, las personas no relacionadas a la odontología toleran desviaciones de hasta ${ }^{3} \mathrm{~mm}$ en el lado derecho.

También se encuentran diferencias según las nacionalidades y grupos étnicos de los evaluadores. McLeod ${ }^{4}$ en el 2011 comparó resultados sobre percepción estética dental entre estadounidenses y canadienses, y encontró que los canadienses fueron más críticos que los estadounidenses al evaluar. Otras evaluaciones incluyeron el atractivo facial. Chang ${ }^{2}$ en el 2011 evaluó la línea media, junto con otras variables de la sonrisa, según el atractivo facial y el género del modelo. Chang encontró que el género no influyó, no encontró diferencias entre las evaluaciones vertidas por los evaluadores femeninos y masculinos.

El nivel de educación y preparación es un factor que también se ha tomado en cuenta en algunas investigaciones. España ${ }^{19}$ en el 2014, evaluó la percepción de estética de sonrisa y sus alteraciones en estudiantes de odontología de diferentes años académicos, y obtuvo que no influye el nivel de preparación con respecto a los resultados.

El grupo racial del modelo y evaluadores influye poco en la percepción estética. Machado ${ }^{10}$, en el 2013, realizó su estudio empleando como modelos las fotografías de dos mujeres, una de rasgos caucásicos y una de rasgos afrodescendientes; realizó la evaluación de una variable distinta (bordes incisales), pero como conclusión, no obtuvo diferencias en cuanto al color de piel de las modelos sometidas a evaluación. En el presente estudio se usó un solo sujeto modelo: mestizo, cuya fotografía y modificaciones fueron sometidas a grupos evaluadores peruanos (ambos grupos).
Otra variable que ha sido incluida en algunos estudios junto con la posición de línea media es el biotipo facial. Whilliams ${ }^{5}$ en el 2014 realizó un estudio de percepción de línea media en personas comunes según biotipo facial, y encontró el valor promedio de $2,92 \mathrm{~mm}$ de aceptación de línea media, y que el tipo europrosopo y el sexo masculino obtuvieron mejor aceptación al ser evaluados.

Las diferencias de edades en los grupos evaluadores, en la presente investigación, sería un factor limitante, pues pudo generar algún sesgo en la percepción.

\section{Conclusiones}

Los ortodoncistas fueron más críticos al mostrar sus percepciones con respecto a las desviaciones de línea media de la sonrisa en comparación con los individuos no relacionados a la odontología, lo que se evidencia en la menor tolerancia en sus percepciones estéticas de la estética de las desviaciones mayores de línea media de sonrisa.

Existen diferencias en las percepciones según el lado derecho o izquierdo en el que se encuentran posicionadas estas desviaciones.

Se encontró que las desviaciones presentadas hacia el lado derecho fueron más identificadas y menos aceptadas que las presentes en el lado izquierdo.

La aceptación de desviación hacia la derecha del sujeto fue de $2 \mathrm{~mm}$ y de $3 \mathrm{~mm}$ hacia la izquierda del sujeto, por ambos grupos evaluadores.

Para ambos grupos evaluadores, los resultados de las desviaciones de 1, 2, 3 y $4 \mathrm{~mm}$ hacia la derecha y de $1 \mathrm{~mm}$ hacia la izquierda fueron estadísticamente significativos mientras que los resultados de las desviaciones hacia la izquierda de 2, 3 y $4 \mathrm{~mm}$ y posición centrada no lo fueron.

\section{Referencias bibliográficas}

1. Peck H, Peck S. A concept of facial esthetics. Angle Orthod 1970;40(4):284-318.

2. Chang CA, Fields HW Jr, Beck FM, Springer NC, Firestone AR, Rosenstiel S, Christensen JC. Smile esthetics from patients' perspectives for faces of varying attractiveness. Am J Orthod Dentofacial Orthop 2011;140:e171-e180.

3. Sarver DM. Soft-tissue-based diagnosis \& treatment planning. Clinical Impressions. 2005;14(1):21-6.

4. McLeod C, Fields HW, Hechter F, Wiltshire W, Rody W Jr, Christensen J. Esthetics and smile characteristics evaluated by laypersons. Angle Orthod. 2011;81:198205.

5. Williams RP, Rinchuse DJ, Zullo TG. Perceptions of midline deviations among different facial types. Am J Orthod Dentofacial Orthop 2014;145(2):249-255.

6. Janson G, Branco NC, Fernandes TM, Sathler R, Garib D, Lauris JR. Influence of orthodontic treatment, 
midline position, buccal corridor and smile arc on smile attractiveness. Angle Orthod. 2011;81:153-161.

7. Springer NC, Chang C, Fields HW, Beck FM, Firestone AR, Rosenstiel S, Christensen JC. Smile esthetics from the laypersons's perspective. Am J Orthod and Dentofacial Orthop. 2011;139:e91-e101).

8. Tupinambá RC, Magnani R, Candido MM, Batista OO Jr. The perception of smile attractiveness. Angle Orthod. 2009;79:634-639.

9. Pinho S, Ciriaco C, Faber J, Lenza MA. Impact of dental asymmetries on the perception of smile esthetics. Am J Orthod Dentofacial Orthop. 2007;132:748-53.

10. Machado AW, Moon W, Gandini LG Jr. Influence of maxillary incisor edge asymmetries on the perception of smile esthetics among orthodontists and laypersons. Am J Orthod Dentofacial Orthop. 2013;143:658-664.

11. Zhang K, Huang L, Yang L, Xu L, Xue C, Xiang Z, Zhao M, Li S, Bai Y, Bai D. Effects of transverse relationships between maxillary arch, mouth, and face on smile esthetics. Angle Orthod. 2015;0(0): 3. Disponible en: http:/www.angle.org/doi/pdf/10.2319/101514.1

12. Jayalakshmi NS, Ravindra S, Nagaraj KR, Rupesh PL, Harshavardhan MP.Acceptable Deviation between Facial and Dental Midlines in dentate population. J Indian Prosthodont Soc. 2013;13(4):473-477.
13. Thomas M, Reddy R, Reddy B J. Perception differences of altered dental esthetics by dental professionals and laypersons. Indian J Dent Res. 2011;22:242-7.

14. Kokich VO, Kokich VG, Kiyak HA. Perceptions of dental professionals and laypersons to altered dental esthetics: asymmetric and symmetric situations. Am J Orthod Dentofacial Orthop. 2006;130(2):141-51.

15. Cardash H, OrmanierZ, Laufer B. Observable deviation of the facial and anterior tooth midlines. J Prosthet Dent. 2003;89(3):282-285.

16. Arillaga JM. Fundamentos de la composición visual. UNED. España. Disponible en: http://www.uned.es/ ntedu/espanol/master/primero/modulos/teoria-de-la-representacion/fundamentos-composicion.htm

17. Bergaondo J. ¿Por qué miramos más el lado derecho de una foto?. Quo. España. 2015. Disponible en: http:// www.quo.es/ser-humano/por-que-miramos-mas-el-lado-derecho-de-una-foto

18. Witt M, Flores-Mir C. Laypeople’s preferences regarding frontal dentofacial esthetics: periodontal factors. J Am Dent Assoc. 2011;142(8):925-937.

19. España P, Tarazona B, Paredes V. Smile esthetics from Odontology students' perspectives. Angle Orthod. 2014;84(2):214-224. 